\title{
A stability routing protocol for the Solar Blind Ultraviolet Ad-Hoc Network
}

\author{
Juan Yang ${ }^{1, a}$, Jianlu Luo ${ }^{1, b}$, Fang Cheng ${ }^{2, c}$ Dandan $\mathrm{Fu}^{1, \mathrm{~d}}$ \\ ${ }^{1}$ Department of Electronic Technology, Officers College of CAPF, Chengdu, 610213, China \\ ${ }^{2}$ Network Management Center, PLA.Chongqing Communication Institute, Chongqing,400030, \\ China

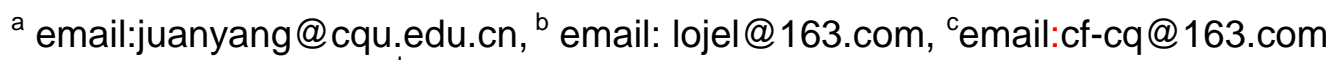 \\ demail:chenchen-008@sohu.com
}

Keywords: Ultraviolet; Ad-Hoc Network; Stability

\begin{abstract}
In this paper, we propose a stability routing protocol to support the stable routing for the solar blind ultraviolet Ad-Hoc network, and to guarantee the efficiency of routing process. The performance simulation analysis confirms the availability and superiority of our routing protocol.
\end{abstract}

\section{Introduction}

The ultraviolet (UV) communication in the solar-blind region is a way to transmit information through the atmospheric scattering, with some advantages, such as, non-line-of-sight (NLOS) transmission, good secrecy, strong anti-interference ability, and low cost of construction and maintenance [1]. With these advantages, the UV communication is popularly used in the solar blind region as a good enough substitute for the radio communication, and widely used in the country, the city outdoor environment and the military communication [2]. But the near atmospheric molecules and particles have a strong absorption effect on the solar blind UV light. As a result, the transmission distance is limited.

The wireless Ad-hoc network is a distributed network temporarily without center, where the nodes can arbitrarily move and all nodes are identical to each other. The source node can get to the destination node through multi hops. However the solar-blind UV NLOS Ad-hoc communication network can be combined with NLOS, strong anti-interference ability, good security of UV transmission and the multi-hop feature of Ad-hoc network to achieve reliable long-distance transmission, which offsets the limitation of the solar blind UV NLOS communication system and the short communication distance. Actually, the solar blind UV NLOS Ad-hoc communication network now becomes a new effective method to satisfy the communication requirements in the various mobile scenarios.

Characterized by a typical distributed system, the topology in the solar blind UV NLOS Ad-hoc Networks varies dynamically and massively. A large number of distributed nodes share the limited channel resources, giving rise to an intensified competition and thus a steep decline in network performance. Accordingly, studies on the stability routing protocol in a distribute-network model is of crucial significance on its applications.

At present, the research on the solar blind UV NLOS communication is mainly concentrated on the effective node coverage range and channel model [3-8], path loss [9] and the modulation and demodulation [10]. There are very few researches on the routing protocol, and there is no research on the routing protocol for the solar blind UV NLOS Ad-hoc communication network.

In this paper, according to the conditions of network and nodes, several characteristics of node stability are taking into account, and a dynamic adaptive stability routing protocol is achieved by perceiving the distributed dynamic network topology with the use of multi-feature fusion and GRF-MAP method. 


\section{Stability Node}

Regarding the node stability in a solar blind UV NLOS Ad-hoc Network, several characteristics are selected to make a fusion; namely, the interestingness of a node to act as a stability node, the remaining battery power, available memory and movement velocity of a node, combined with the degree to be a key node. All of these can measure the stability of a node, with the major reasons listed as follows.

(i) The remaining battery power of a node (P). The stability nodes should take on extra responsibility, and therefore their battery power consumptions far exceed the normal nodes'. The nodes with more remaining battery power should be given priority to be the stability nodes.

(ii) Available memory of a node. Due to the fact that the stability nodes should take the responsibilities to maintain the communications, the nodes with a large memory are more suitable for the stability nodes.

(iii) Movement velocity of a node. To avoid a declining network stability induced by the frequent changes of stability nodes, the nodes with lower movement velocities are more inclined to be the stability nodes.

(iv) The degree to be a key node. In a solar blind UV NLOS Ad-hoc Network, some edge nodes connected two or more regions alone are more likely to appear. These nodes exhibit more power consumptions. Once they are out of work, the network's topology will be partitioned and thus the communication is interrupted. The node satisfies a relationship $\mathrm{Ni}-\mathrm{Mi} \geq 2$ can be defined as a key node, in which $\mathrm{Ni}$ and $\mathrm{Mi}$ are the node's neighbor degree and basic circuit degree, respectively. Moreover, the node whose key-node degree is greater than or equal to 2 can be regarded as the stability node.

\section{Construction of a prior energy function}

In accordance with the detection results in the initial label field, the nodes in a solar blind UV NLOS Ad-hoc Network can be divided as the stability nodes and the general nodes, respectively, and then they obtained binarization results are denoted as $\hat{f}_{k}(\bullet)$.

$$
\hat{f}_{k}(\bullet)= \begin{cases}0 & \text { label stability node for node } \mathrm{i} \\ 1 & \text { label general node for node } \mathrm{i}\end{cases}
$$

In which the prior energy function $U$ (f) is the related energy in the initial label field. Given a label field $\mathrm{f}(\mathrm{)})$, the detection results at k-th time. $\hat{f}_{k}(\bullet)$ are treated as the initial label field.

Essentially, the potential function $\mathrm{Vc}(\mathrm{f})$ mirrored the dependence of the center node to its neighbor. The better the state of center node fits with the neighbor, the lower the potential $\operatorname{Vc}(\mathrm{f})$ is. The prior potential function is then constructed as follows,

$$
V_{c}\left(f_{k}(i)\right)=\frac{f(i) \neq f_{k}(j)}{\# C_{i}}
$$

in which $\# \boldsymbol{C}_{i}$ denotes the number of nodes which includes node i as well. Since the number of neighbors in the neighborhood system of a center node is different, serves as a normalization factor in the construction of the prior potential function. Moreover, is the label of the neighborhood system, $\mathrm{j}$ is the one-hop neighbor with node $\mathrm{i}$ in the $\mathrm{Ci}$, and $f_{k}(j)$ is the label of node $\mathrm{j}$ at $\mathrm{k}$-th time.

In line with the definition of energy function, the prior energy function of node $i$ at $k$-th time can be described as

$$
U(f)=\sum_{f_{k} \in L} V_{c}\left(f_{k}(i)\right)
$$

in which $\mathrm{L}$ represents the set of labels of the neighborhood system which includes node i. When $\mathrm{f}$ is consistent with all nodes or most of nodes in the neighborhood system, $U(f)$ turns lower, and the system can achieve a favorable consistency effectively. Additionally, due to the introduction of the normalization factor, the nodes with fewer neighbors can acquire a larger weight, contributing to a 
consistency of the system. $\mathrm{U}(\mathrm{f})$ is a data-driven item induced by the initial label, reflecting the consistency between the prior label and the required label. When the required label fits well with the prior label, the energy of this item reduces otherwise it increases.

\section{Construction of a likelihood energy function}

The likelihood energy function is referred to as the corresponding energy functions of the correlation between the nodes in the label field under the constraint conditions. The acquired multi-feature information are used as the constrain conditions, and the key features to constrain the node stability are then converted to the probability of each feature's occurrence. Due to the fact that the selection of the key characteristics of node stability is independent, i.e., the conditions are independent; the likelihood function can be expressed as

$$
P(X \backslash f)=P\left(x_{p}, x_{M}, x_{V}, x_{D} \backslash f\right)=\prod_{j=1}^{5} P\left(x_{j} \backslash f\right)
$$

The analysis results in Section Node stability indicate that $\mathrm{xV}$ and $\mathrm{xD}$ should be minimized for the maximization of node stability, and thus Eq. (4) can be written as

$$
\max P(X \backslash f)=\max \frac{P\left(x_{p} \backslash f\right) P\left(x_{M} \backslash f\right)}{P\left(x_{v} \backslash f\right) P\left(x_{D} \backslash f\right)}
$$

Taking a positive logarithm on Eq. (5), we can obtain

$$
\min [-\ln P(X \backslash f)]=\min \left[-\ln P\left(x_{p} \backslash f\right)-\ln P\left(x_{M} \backslash f\right)+\ln P\left(x_{v} \backslash f\right)+\ln P\left(x_{D} \backslash f\right)\right]
$$

Therefore, the defined likelihood energy function is given by

$$
U(X / f)=U^{p}+U^{M}+U^{V}+U^{D}
$$

In which

$$
U^{p}=-\ln P\left(x_{p} \backslash f\right), U^{M}=-\ln P\left(x_{M} \backslash f\right), U^{V}=\ln P\left(x_{v} \backslash f\right), U^{D}=\ln P\left(x_{D} \backslash f\right) .
$$

\section{Estimation of the probability density of the components in the stability vector}

According to the Linderberg-Levy theorem, combined with the experimental results in a large number of literatures, the nodes' movement velocity, degree and the available memory are assumed to follow a Gaussian distribution, i.e., $x_{V} \sim N\left(u_{V}, \sigma_{V}{ }^{2}\right), x_{D} \sim N\left(u_{D}, \sigma_{D}{ }^{2}\right), x_{M} \sim N\left(u_{M}, \sigma_{M}{ }^{2}\right)$; Besides, $\mathrm{xp}$ is exponentially distributed with a probability density $\mathrm{p}(\mathrm{pi})$ in the following forms $p\left(p_{i}\right)= \begin{cases}\lambda e^{-\lambda p_{i}} & p_{i}>0 \\ 0 & p_{i}<0\end{cases}$

In the networks initial state, any node in the network broadcasts a 'Hello' message (the energy is measured with the node' movement velocity as the index). Then the neighborhood system of node $\mathrm{i}$ is identified, in which the node with the minimum velocity is labeled as ' 0 ', and the other nodes are labeled as ' 1 '. Finally, all the nodes in the network are labeled. Each node broadcasts a 'Hello' package, and sends its label to the neighbor nodes. The stability energy of the neighborhood system is calculated according to Eq. (7). After calculation, the node with the minimum energy is taken as the stability node, labeled as ' 0 '.

The main idea of the stability routing protocol for each node determining itself to be the forwarding node to participate into the routing process is as follows: the node, which is labeled as ' 0 ', and with the largest stability value, has the highest opportunity to be the forwarding node.

The stability routing protocol is constructed as follows.

The route request process works as follows.

Step 1: The source node starts route request by broadcasting "Route Request" message which could be received by each one-hop neighbors and labeled as ' 0 '.

Step 2: After receiving "Route Request” message, each one-hop neighbor and labeled as ' 0 ' node checks whether it is the destination node or not, or whether it has a valid route to the destination or not. If it is, the process goes to step 5; otherwise, the process goes to step 3. 
Step 3: Each one-hop intermediate neighbor and labeled as ' 0 ' node waits for time Ti.

Step 4: The first node that runs out the time Ti rebroadcasts the "Route Request" message. The node that hears the same "Route Request" message more than once ignores it and stops the time Ti waiting. Then, the process goes to step 2 to continue the relaying of the "Route Request" message until the "Route Request" message reaches the destination.

Step 5: When the destination node receives the "Route Request" message, the route request process ends and the route reply process starts; otherwise, the process goes to step 2 to continue the relaying of the "Route Request" message until the "Route Request" message reaches the destination.

The route reply process works as follows.

Step 1: When the "Route Request" message reaches the destination node D or an intermediate node that knows a valid route to node $\mathrm{D}$, the "Route Reply" message is sent to the source node $\mathrm{S}$ along the inverse accumulated path. The "Route Reply" message includes inversed full path that the "Route Request" message traversed along.

Step 2: Nodes on the path relay the "Route Reply" message to the next-hop node toward the source node S, until the "Route Reply" message reaches the source node S.

\section{Test results}

Using the corresponding underlying codes of NS2, the stability routing protocol in the present work was implemented, and we show that our protocol performs better than DSR.

Table 1 Parameter for simulation of The stability protocol (STP)

\begin{tabular}{c|c}
\hline parameter & values \\
\hline number of nodes & 20 \\
The simulation range & $1000 \mathrm{~m} \times 1000 \mathrm{~m}$ \\
Packet Size & $512 \mathrm{bit}$ \\
simulated time & $1000 \mathrm{~s}$ \\
Maximum speed for node & $20 \mathrm{~m} / \mathrm{s}$ \\
Mobility model & RWM \\
Traffic type & CBR (UDP) \\
\hline
\end{tabular}

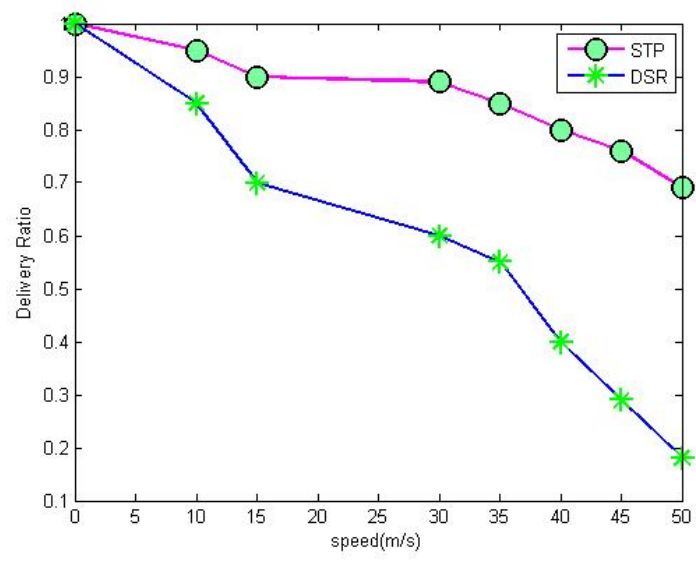

Fig. 1. Delivery ratio vs. speed.

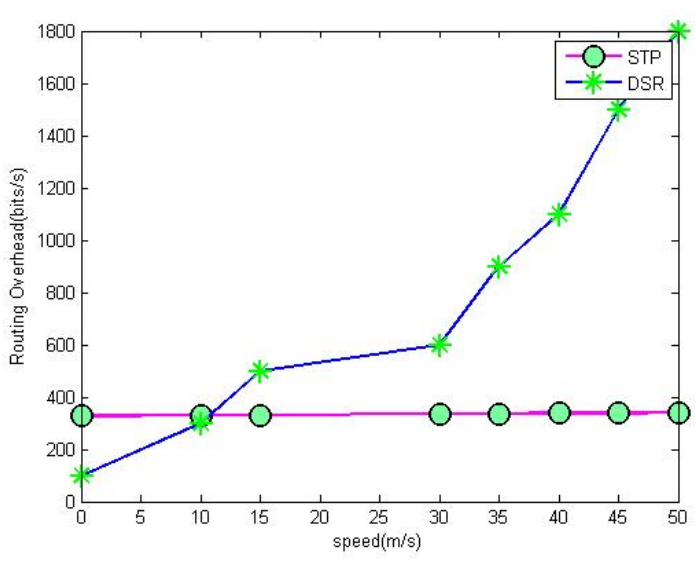

Fig.2. Routing overhead vs. speed.

As shown in the figure 1, the delivery ratio is much higher than that of DSR, and with the increase of speed, the performance of STP is relative stable. Then, as shown in the figure 2, the routing overhead is much smaller than that of DSR, and with the increase of speed, the performance of STP is relatively stable.

\section{Conclusion}

In this paper, we propose a routing protocol, named as STP, in the solar blind UV NLOS Ad-hoc Network considering stability metric. We choose the node with high stability value to attend the routing process. By doing this, the path between source node and destination node could be relatively stable. Finally, by simulation, 
we conclude that STP performance is better than that of DSR.

\section{Acknowledgement}

In this paper, This work was supported in part by a grant from the National Science Foundation of China under Grant No. 61173131, Based and advanced research projects of Chongqing of China (Grant No. cstc2013jcyjA40033), The special funding for Scientific research project Of Chongqing postdoctoral researchers under Grant No. XM20120054.

\section{References}

[1] ZHAO T.F, KE X.Z LASER JOURNAL, 31(6) (2010), 40.

[2] Chou W “Research on MAC Protocols for Wireless Mesh Networks Using Directional Antennas,” M.S. Thesis, Xi dian University,Xi’an,China,2007

[3] Li, Y., Wang, L., Xu, Z., Krishnamurthy, S. V. IEEE journal on selected areas in communications: Special Issue on Advances in Military Networking and Communications. (2011), 135

[4] Xu Z., Sadler, B. M. IEEE Communications Magazine, (2008).46(5).

[5] Wang J L, Luo T, Dai M. UV NLOS communications atmospheric channel model and its performance analysis. Los Angeles: World Congress on Computer Science and Information Engineering,(2009),p.85

[6] Feng T, Xiong F, Chen G, Fang Z.J, Optic 11(9)(2008) 612

[7] Zhang H.L, Yin H.G, Jia H.H, Yang J.C, Chang S.G, Optics Communications, 28(5)(2012), 1771

[8] Chen G, Abou-G, Xu Z.Y. Optics Express,16(19)(2008),15059

[9] He Q F,Sadler B M,Xu Z Y.”Modulation and Coding Tradeoffs for Non-line-of sight Ultraviolet Communication” Pro. of SPIE,H-1-12(2009)7464.

[10] KE X.Z, He H， Chen X. Ournal of Optoelectronics. Lase 21(7)(2010), 1002 